\title{
The Design and Implementation of Campus Shopping Site Based on User Experience
}

\author{
Yun LIU \\ AnHui XinHua University, School of Information Science and Technology, HeFei 230088
}

Keywords: User experience, E-commerce, Design of usability

\begin{abstract}
With the rapid development of new e-commerce model, the implement the retail model of online to offline has increasingly high requirements to users experience. This paper combines the users experience theory model with the Business Logic Layer design of shopping site, proposes the "user center" ideal to design and implement the campus shopping site, and adopts the concrete technology to implement all functional modules.
\end{abstract}

\section{Introduction}

The campus $\mathrm{C} 2 \mathrm{C}$ model is an e-commerce modelfor students transaction in universities. A website with good user experience will gain many more users, and even bring lots of loyal customers to improve the number of active users and page views. Combined with the analysis of structure layer, profile layer and presentation layer in the James Garrett user experience model, Campus Shopping Site designs the retail channel of online and offline interactive marketing, and also promotes custom-centric ideal to complete the implementation of the product function and interface design, in order to enhance shopping convenience.

Quality of user experience is the resulting from interactions of the network layer and the service layer. Set Enas the contribution of the network layer to quality of user experience, $N_{1}, N_{2} \ldots N_{m}$ as the network layer index, $f$ as mapping relation:

$$
\mathrm{En}=\mathrm{f}\left(\mathrm{N}_{1}, \mathrm{~N}_{2}, \ldots, \mathrm{Nm}\right)
$$

Set $E_{i j}$ as the contribution of the first class $i$ business service layer to quality of user experience, $\mathrm{S}_{\mathrm{ij}}$ as the service layer index in first class $\mathrm{i}$ business, $\mathrm{j}$ as the number of indexes in first class $\mathrm{i}$ business, $\mathrm{g}$ as mapping relation:

$$
E_{\mathrm{ij}}=\mathrm{g}\left(\mathrm{S}_{\mathrm{i} 1}, \mathrm{~S}_{\mathrm{i} 2}, \ldots \mathrm{S}_{\mathrm{ij}}\right)
$$

Set $\mathrm{Ki}$ as the scale in first class $\mathrm{i}$ business, and $\sum_{i} K i=1$, Es as the contribution of the first class $\mathrm{i}$ business service layer to quality of user experience, then:

$$
\mathrm{E}=\sum_{i} K i \cdot \mathrm{E}_{\mathrm{ij}}
$$

Set $\mathrm{E}$ as the integral quality of user experience in mobile networks, then:

$$
\mathrm{E}=\mathrm{En} \cdot \mathrm{Es}
$$

The integral quality of user experience in mobile networks can be described as the product of network layer quality and service layer quality. The network layer quality should meet or exceed a certain threshold in order to meet the basic needs of users. Due to a wide range of the service, frequency of use, and the different in service time, the evaluation of service layer quality will affect user experience directly. According to the information organization, labeling, navigation and search, the service layer can improve the information availability and good user experience. The design of information organization should try to achieve accessibility and intelligibility, and then find the target information effectively from a large amount of information collection. 


\section{Availability}

\section{users needs}

Based on integration information resources faces users directly, Campus Shopping Site highlights demand and services, coordinates the users demand, the service organizations strategy and environmental requirements, then shifts to the design stage in user experience building. In order to provide users clear and understandable information, interface design is on the basis of information architecture. Arranging the interface elements reasonable and distinguishing the degree of important information will implement the human-computer interaction easily. This platform faces WEB service set for buyers and sellers, accesses the site processing services, and makes goods inquiry in timely, order status and shopping links evaluation through mobile devices and PC. The system apply network programming technology (ASP.NET), database software applications (Microsoft SQL Server 2008), C \# and other technologies synthetically to implement the construction of the trading

platform.UI layer using Bootstrap framework responsive design, BLL layer uses Ajax client request $\mathrm{s}$ and return results and DAL layer data using Entity Framework.

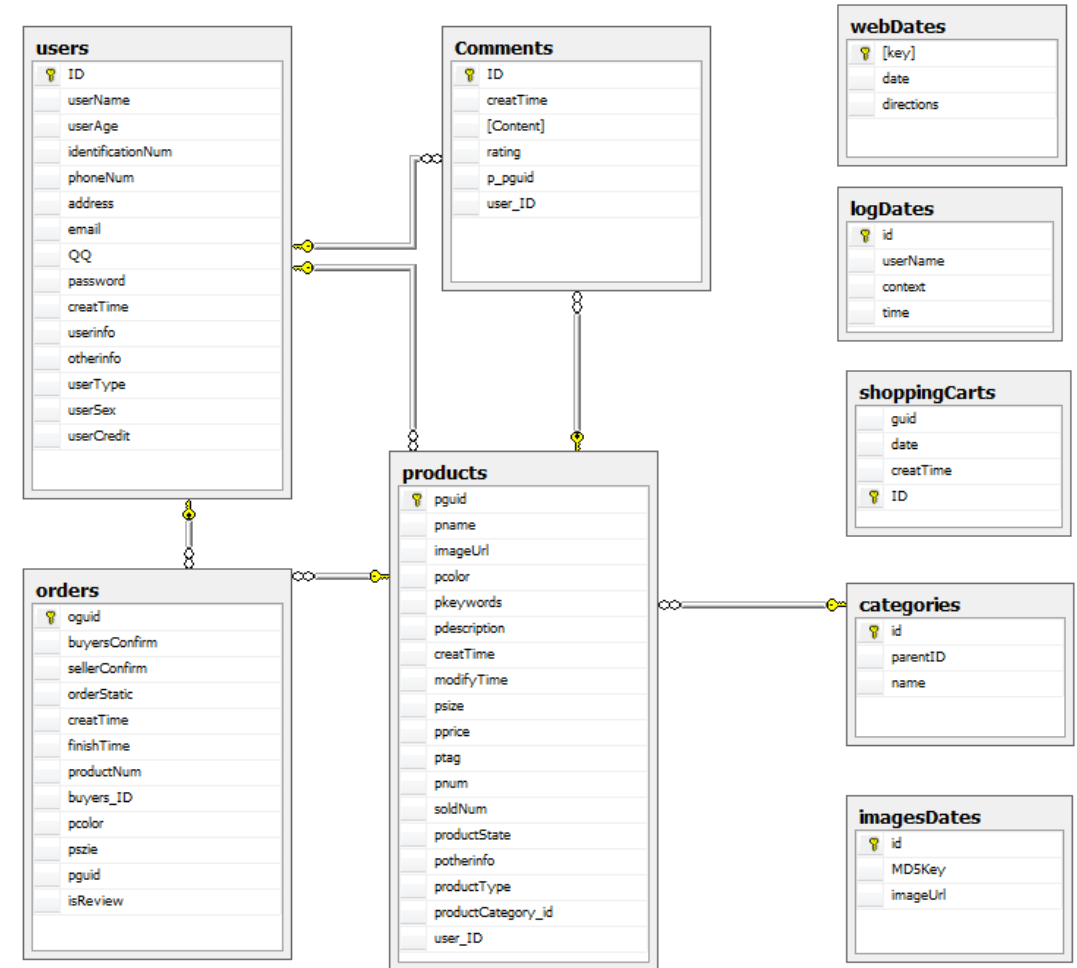

Figure 1 Database Diagram

There are nine tables, including user table, the orders table, merchandise table, comment table, commodity classification table, image data table, cart tables, the log table, and the configuration table. The relationships are shown as above Figure 1.

\section{Interface Design of High User Experience}

The user inputs user name to sign into the site. Each time he inputs a character, the system will judge whether the current user name exists in the system, then feedback to the user timely, to reduce the possibility of failure login and registration. After the user logs on, the site will store the user login information in the cookie, and save for three days, to reduce repetition logging in. The main code is shown in Figure 2: 


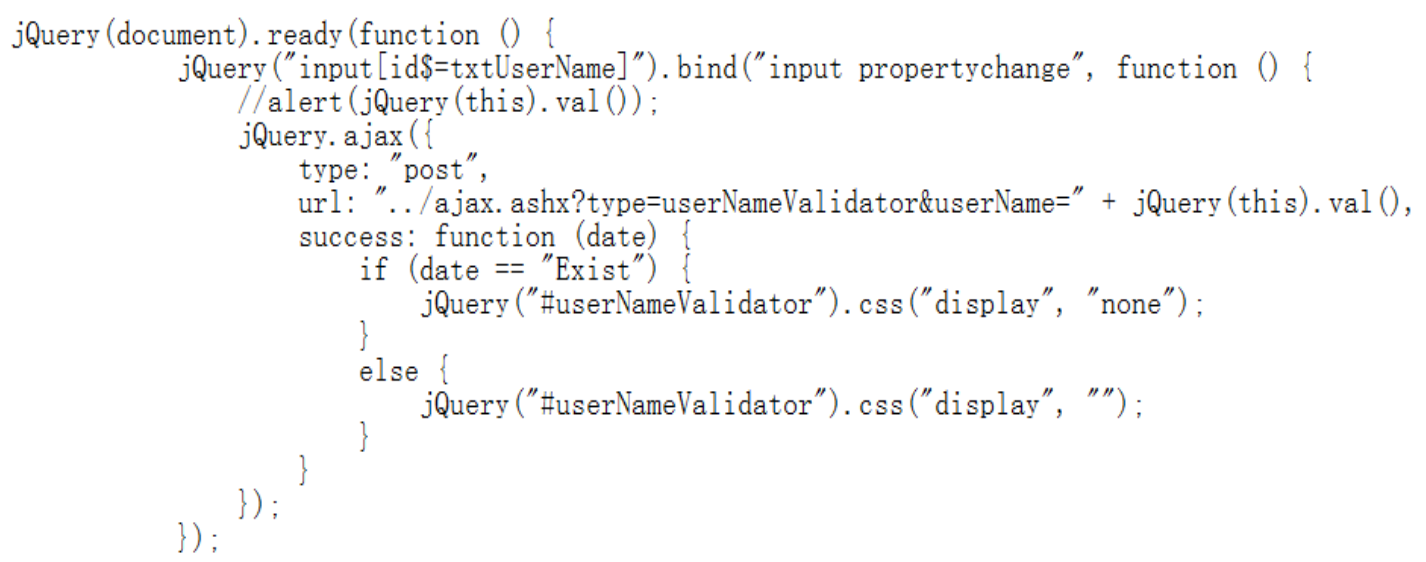

Figure 2 User Name timely verification code

Rational interface layout will reduce the user attention dispersion, and also highlight the view point. In the main interface system, an icon or menu are usually layout at the top, bottom and horns of the viewing area, to reduce user attention dispersion. The icon design follows the conventionalized and simple rules to reduce the cognitive work of users, and also improves the cognitive efficiency. The forepart plays an important role in user experience. Due to the screen of mobile terminal is too small, then the page content are stacked together, so it's difficult for the users to click the links correctly, and the user experience is so poor. If using the Bootstrap framework in forepart, the page content will be adjusted according to the screen width of different devices. So the framework needs the following code on each page $<$ head $>$ :

$<$ Link href $=$ "../ style / bootstrap.min.css" rel = "stylesheet" type $=$ "text/ Css "/>

$<$ Script $\mathrm{src}=$ "../ js / bootstrap.min.js" type $=$ "text / javascript" $></$ script $>$

In order to meet the different hobbies of users to the site color,the whole site color is off white and adds a floating button on the right to switch all 16 kinds of colors, mainly throughloading different CSS stylesheet to control the site's overall colors, which means loading different CSS style sheetsto choose different colors. The main codes are as following:

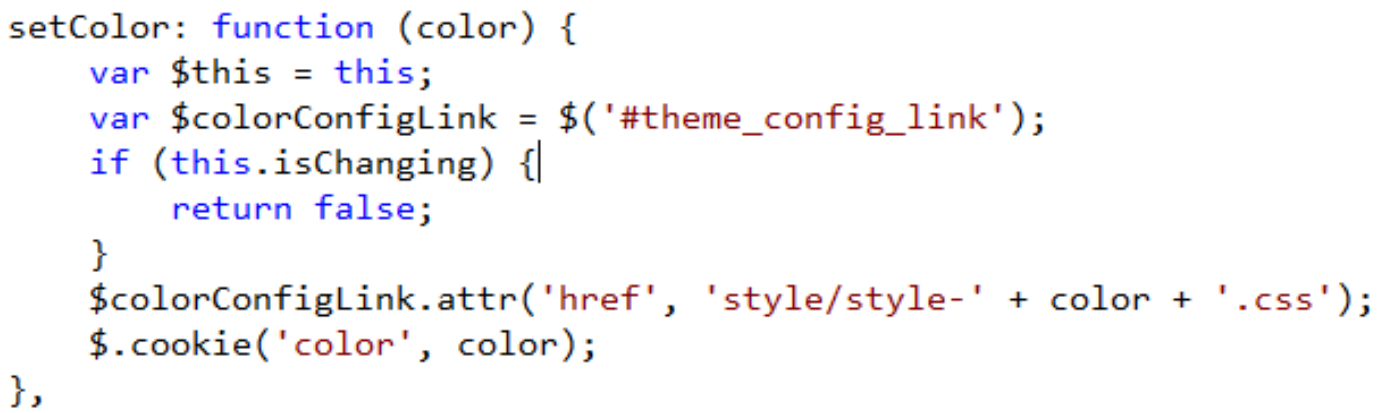

Figure 3 Code for switchingcolor

When users browse the merchandise, placing the mouse on the goods will shade and highlight the important information of this kind to avoid the users choosing wrong goods. When users fill in the information in the current text box, using glowing effect in the frame to allow the users to know where they are filling timely. So the Hover effect highlights the important information in the user interface design and helps to enhance the shopping experience.

3Optimizing the Details in Presentation Layer

\section{Implement Asynchronous Image Upload}

There will be lots of product images in Shopping platform pages, which affects the loading speed of the site inevitably. In the data storage section, in order to reduce the size of the database, the productimages are stored only by one path, rather than store them in the database, which will 
speed up reading the data of speed product images. In upload section, use Ajax and HTML5 technologiescan implement multi-products images asynchronous uploading and after that, it also displays on the page timely. In the background section, based on the uploading images, the system will produce a MD5 check code, which will be the basis of checking the image whether exists in the server. If any, the system will not store the image but return to the address of the image. If not, the system will compress the image, then sent the image path and the corresponding MD5 into the database for the next match. This can reduce the image size effectively, speed up the page loading, while unifiesall image size, and makes a better effect for the page display. The main code is shown in Figure 4:

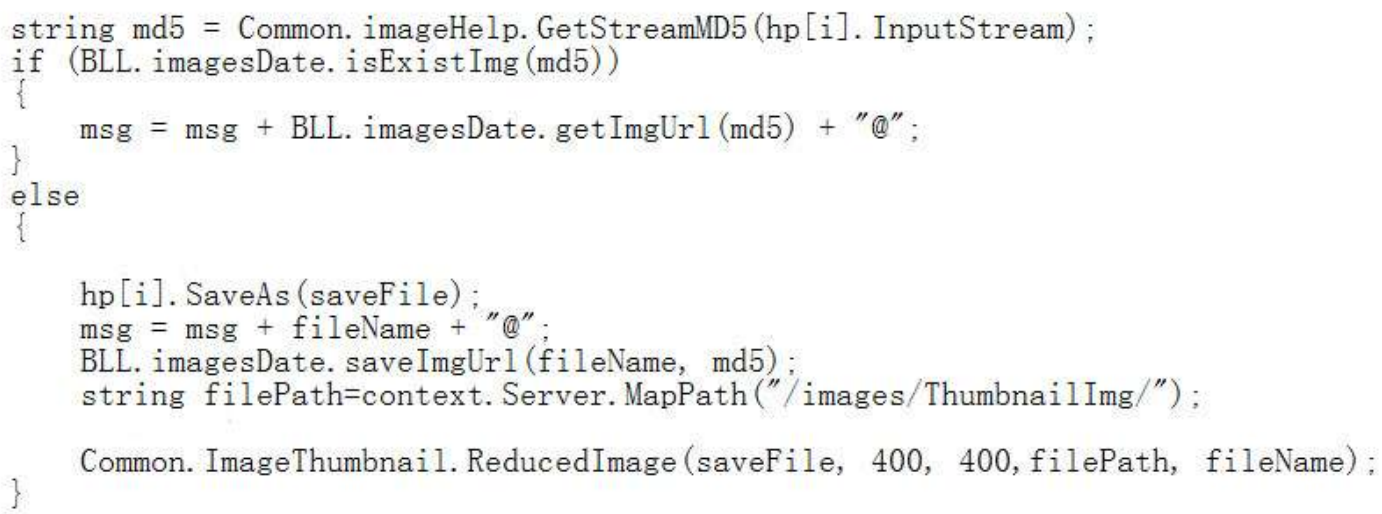

Figure 4 The Core Code for Processing the Product Image

\section{Optimizing the Shopping Cart Design}

On each top of the page, there will be a shopping cart icon, which displays the products that user chose. Clicking the icon will show animation effects, the whole page will move to the right, and on the left there will be a shopping cart details. Using jQuery UI technology to avoid the page hopping during the inquiry procedure, reduce the loading time, and contribute to respond the shopping cart information timely. When the user modifies the merchandise stock, clicking the merchandise stock will popup a dialog box to fill in the remaining products number, if users input the remaining products number, the system will finish all other related information automatically. However, the shopping cart in traditional shopping site often uses Session for storage, which leads to the related shopping cart information loss if the users close the current window or stay long time. In that case, the users have to try it again and get a poor experience. Therefore, in order to avoid this problem, during design the site, using cookie plus the database to store the related shopping cart information. Whether the user closes the browseror stay for a long time or not, the shopping cart information will still be still saved. In addition to this, the system also encrypts shopping cart information to protect the user's privacy.

\section{Analysis and Design of the Navigation Function}

During the shopping process, in order to screen the merchandise, the system choose to design the navigation bar, which improves the user experience greatly. There is a navigation bar at the top of the product display page, and the displays are different depending on the classifies selected by the user.The whole page adopts Ajax technology to implement the screen and paging and updates the product list box which the users choose, but keep the others unchanged, which reduce the page skip and the waiting time.It will make the operating system more timely and efficient, and get optimal display results. Commodity data requirement is as following: 


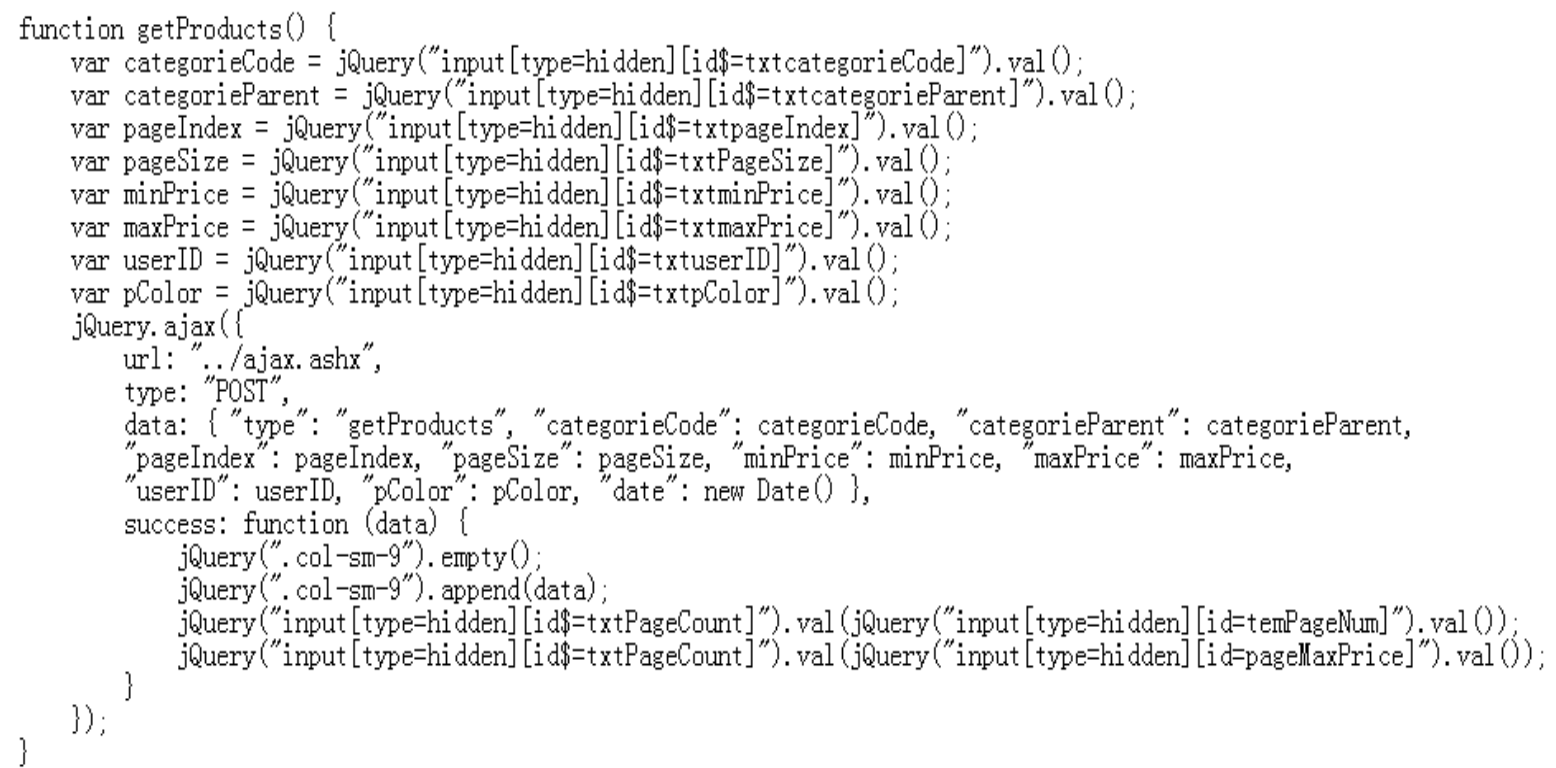

Figure 5 Ajax Require Commodity Data

\section{Test and Analysis}

Under the circumstances of multi-player online shopping, in order to ensure shopping implemented successfully, make certain the loading speed of the whole site page, and insure the system can operate normally during the users is too large, in the final development, the system is tested negative. Using Microsoft Application Center Test for testing, the system selects the home page, category page, product details page and shopping cart page which are the most frequently used to simulate 500 users simultaneously online shopping.

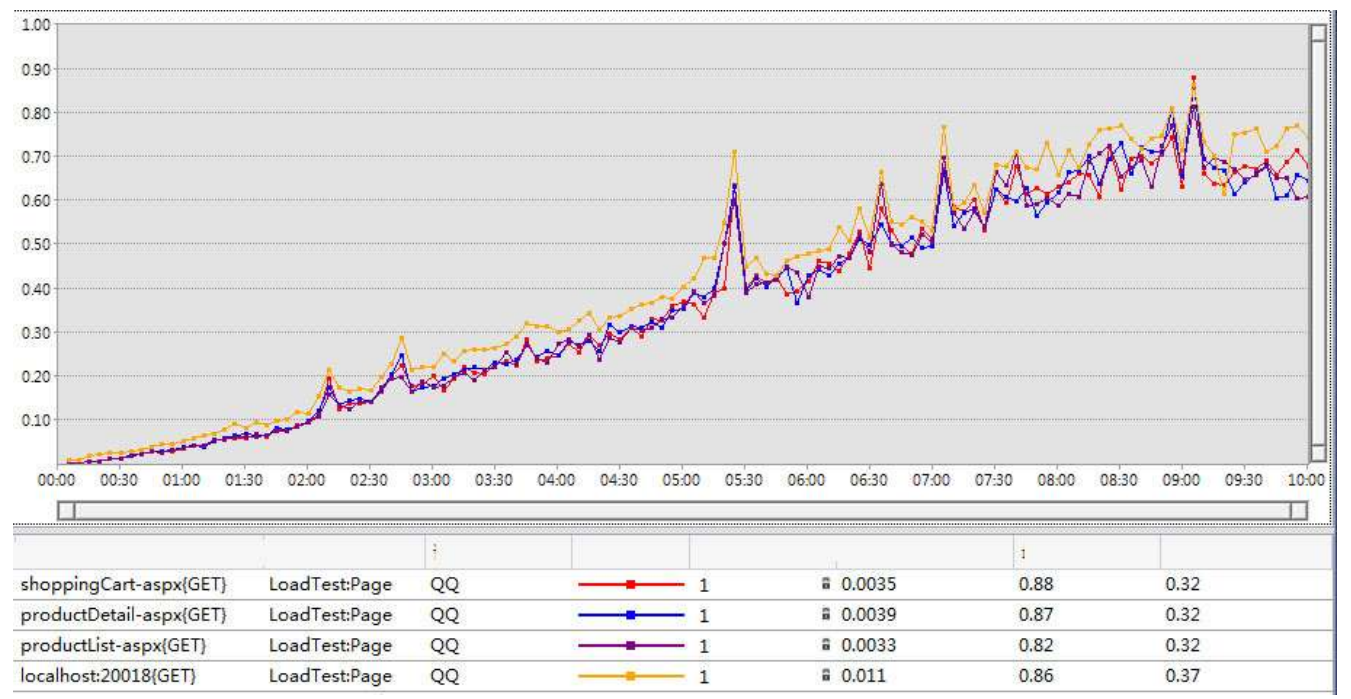

Figure 6 Page Response Time

From the figure 6, when 500 people users simultaneously online shopping, the system can operate and feedback successfully. As the number of users increases, the page response time has also increased and leveling out during 7-8 minutes, not excess of 1 second. The results meet the demand. 


\section{Summary}

This paper adopts ASP, NET + Ajax to develop the shopping platform. UI layer adopts Bootstrap framework responsive design. DAL layer adopts Entity Framework operating data. BLL layer processes client requests and returns results. Those solve the problems of experiencing to gain the massive information between PC and mobile terminal, while speed up the page loading time, and improve the user experience.

\section{Author in brief}

Yun LIU, born in Hefei city of Anhui province in 1983, of Han nationality, master, lecturer, whose research direction is Computer software technology, information technology application. The anhui province quality engineering project (project number: 2014 sxzx021)

\section{Reference}

[1].Wen-bo guo Based on the standardization of user experience research on web design and development process [D]. Shanghai normal university, (2012).35-60

[2] xue-lian li, Yang Zhen, $\mathrm{Mr} \mathrm{Xu}$. C2C e-commerce platform user experience design research [J]. Journal of fine arts, 3(2013) 102-105.

[3] lily Yang. User experience oriented electric shopping website interface design research [D]. East China university of science and technology, (2014)76-80.

[4] Wang Jiang. Campus C2C online trading platform of application research [J]. Journal of information science and technology of China, 5(2010)92-93.

[5] zhao Jin. Hybrid Web application user experience design [D]. The capital economic and trade university, (2014).52-53 